\title{
Standardising the management of alcohol withdrawal at an NHS district general hospital
}

\author{
Authors: Kathleen Turner, Tina Maheswaran and Katherine Dodd
}

\section{Aims}

$>$ To review the management of alcohol withdrawal at the trust.

$>$ To develop an evidence-based policy to standardise the management of alcohol withdrawal.

\section{Methods}

A lack of consistency in the management of alcohol withdrawal was identified at the trust. A multidisciplinary working group was set up to review the existing policies, and evaluate the evidence available. A trust-wide policy was introduced and the Clinical Institute Withdrawal from Alcohol score was used to manage alcohol withdrawal. Three main wards were identified for audit and education - emergency department, medical admissions unit and the gastroenterology ward. A 'spot audit' of all inpatients requiring treatment for alcohol withdrawal on these wards was undertaken before, and ongoing audit has occurred after, introduction of the policy.

\section{Results}

Variation in the management of alcohol withdrawal was found. Three different alcohol withdrawal regimens were in use: two symptom triggered and one 8-day fixed-dose regimen.

The mean number of days patients required chlordiazepoxide was similar before (2.35, range $1-5)$ and after (2.2, range 1-6) the introduction of the new alcohol withdrawal policy. Patients now receive appropriate monitoring and doses of chlordiazepoxide based on the severity of their withdrawal.

An overprescription of chlordiazepoxide in patients with significant comorbidities (alcoholic liver disease, chronic kidney disease or older) was identified. Following the introduction of separate prescription charts for chlordiazepoxide and lorazepam, there has been an increase in the appropriate use of lorazepam. Prior to separate charts, $0 \%$ $(0 / 12)$ of patients who should have been prescribed lorazepam were given lorazepam, compared with $83 \%$ (5/6) following implementation of separate charts.

\section{Conclusions}

This district general hospital now has a robust policy on the management of alcohol withdrawal, with two audits post implementation demonstrating ongoing improvements. There has been an increase in the use of shorter-acting benzodiazepines in patients at risk of becoming over-sedated. Education has been provided to nursing staff and doctors to deal with alcohol withdrawal. 